\title{
The response patterns of infantile spasms to treatments in 156 patients
}

\section{Hormonal therapy with intravenous synthetic ACTH appears promising}

Muhammad T. Alrifai, MD, Ahmad R. Al-Rumayyan, MBBS, FRCPC, Waleed A. Al-Tuwaijri, MBBS, Duaa M. Baarmah, MBBS, Safiyyah A. Asiri, MBBS, Ahmad H. Bali, MBBS, Muatassem A. Alsadhan, Medical student, Shaden H. Alsugheir, Medical student.

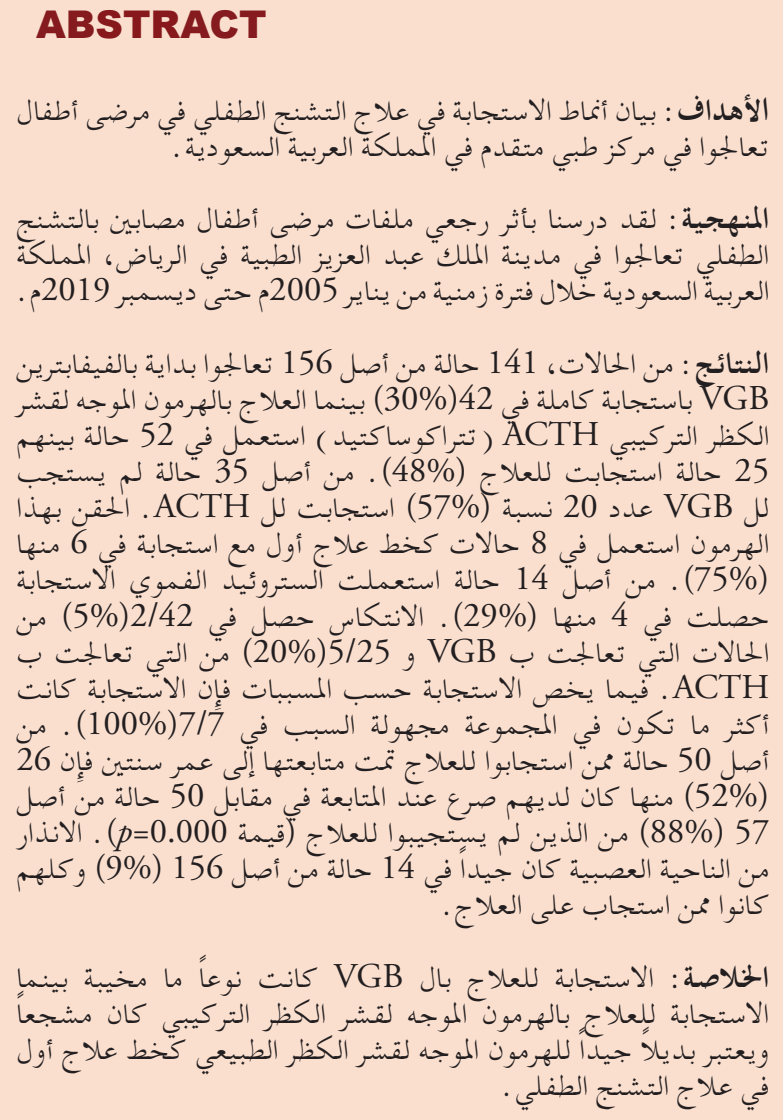

Objectives: To report the efficacy of intravenous (IV) synthetic ACTH (Tetracosactide) in the treatment of infantile spasms.

Methods: This is a retrospective chart review of patients with a diagnosis of infantile spasms conducted at the Pediatric Department of King Abdulaziz Medical City (KAMC) in Riyadh, Saudi Arabia, from 01-012005 to $31-12-2019$.
Results: Of the 156 cases, 141 were treated initially with vigabatrin (VGB) with a complete response seen in $42(30 \%)$. Synthetic ACTH (Tetracosactide) IV injections were used in a total of 52 cases with response in $25(48 \%)$. Of the 35 cases which initially failed with VGB, 20(57\%) responded to synthetic ACTH. The injections were used as a first line in 8 cases with response in $6(75 \%)$. The response to oral steroids was seen in $4 / 14(29 \%)$ cases. A relapse was seen in $2 / 42(5 \%)$ of patients treated with VGB and in $5 / 25(20 \%)$ of those who were treated with synthetic ACTH. The response was highest in the idiopathic group with $7 / 7(100 \%)$. Epilepsy at 2 years was seen in $26 / 50(52 \%)$ and $50 / 57(88 \%)$ of the responders and non-responders, respectively $(p=0.000)$. Only $14 / 156(9 \%)$ of cases had a fair neurological outcome. All of them were from the responder group

Conclusion: The response to VGB is suboptimal, while the response to synthetic ACTH is encouraging making it a good alternative for natural ACTH as a potential first line therapy in infantile spasms.

Neurosciences 2022; Vol. 27 (1): 40-44 doi: 10.17712/nsj.2022.1.20210116

From the Department of Pediatrics (Alrifai, Al-Rumayyan, AlTuwaijri, Baarmah, Bali), Neurology Division, King Abdullah Specialist Children's Hospital, King Abdulaziz Medical City, from the College of Medicine (Alrifai, Al-Rumayyan, Al-Tuwaijri, Baarmah, Asiri, Bali, Alsadhan, Alsugheir), King Saud bin Abdulaziz University for Health Sciences, and from King Abdullah International Medical Research Center (Alrifai, Al-Rumayyan, Al-Tuwaijri, Baarmah, Asiri, Bali), Riyadh, Kingdom of Saudi Arabia.

Received 14th December 2021. Accepted 6th September 2021.

Address correspondence and reprint request to: Dr. Muhammad T. Alrifai, Division of Pediatric Neurology, Department of Pediatrics, King Abdullah Specialist Children's Hospital, Riyadh, Kingdom of Saudi Arabia.E-mail:Rifaim@ngha.med.sa

ORCID ID: https://orcid.org/0000-0002-5203-438X 
Tnfantile spasms is an age-dependent epileptic syndrome that typically presents during the first 2 years of life and has a peak presentation at 4 to 9 months of age. ${ }^{1,2}$ The spasms are brief seizures that come in clusters and are usually associated with developmental arrest or regression. The characteristic electroencephalographic (EEG) pattern is called hypsarrhythmia and has the form of high amplitude multifocal epileptiform discharges and a chaotic high amplitude slow background. ${ }^{3}$ Overall, the prognosis of infantile spasms is unfavorable, with $60 \%$ having epilepsy and $75 \%$ having developmental delay after 2 years of follow-up. 4 Furthermore, this epileptic syndrome has various causes. The International League Against Epilepsy classifies infantile spasms as cryptogenic and symptomatic. Cryptogenic infantile spasms have no identifiable cause with fair development prior to the onset of seizures. ${ }^{5}$ Symptomatic causes of infantile spasms include prenatal causes, such as brain malformations, intrauterine infections, chromosomal anomalies, and single gene causes. ${ }^{5}$ Premature delivery and periventricular leukomalacia are also becoming important causes of infantile spasms with the increased survival of very premature infants. ${ }^{6}$ Postnatal causes, such as hypoxic ischemic encephalopathy and meningitis, are also common and well-identifiable causes. ${ }^{5}$ Hereditary neurometabolic disorders rarely cause infantile spasms and have only been discussed in case reports; however, they accounted for $12.5 \%$ of cases in a study done at our institution. ${ }^{7}$ This was a result of the high rate of consanguineous marriages in the Saudi population. ${ }^{8,9}$

The 2 treatment modalities that showed benefits with this epileptic syndrome include vigabatrin(VGB) and hormonal therapy. ${ }^{10-12}$ The medication that showed the best response rate was ACTH. The response rate varies across studies and is about $50-90 \% .{ }^{13}$ The ACTH was also associated with a better outcome and neurocognitive development. ${ }^{14}$ High dose oral corticosteroid has been advocated as an alternative therapy with a comparable efficacy of $67 \% .{ }^{12}$ However, other antiepileptic drugs that showed some efficacy include valproic acid, topiramate, zonesamide, and nitrazepam. ${ }^{13}$ Early treatments may contribute to better outcomes. ${ }^{15,16}$

The primary goal of this study is to report the efficacy of intravenous (IV) synthetic ACTH (Tetracosactide) in the treatment of infantile spasm patients presenting to a

Disclosure. Authors have no conflict of interests, and the work was not supported or funded by any drug company. tertiary care center in Saudi Arabia. The secondary goal is to report the response to other modalities particularly VGB and oral steroids, as well as reporting outcomes according to responses.

Methods. The study is a retrospective review of all cases in the Pediatric Department at King Abdulaziz Medical City (KAMC) in Riyadh, Saudi Arabia. KAMC is a tertiary care center that serves the National Guard population. The duration of the study was 15 years, from 01-01-2005 to 31-12-2019. The cases were identified through medical records via diagnostic codes. ${ }^{7}$

Cases were included if they fulfilled the criteria of diagnosis of infantile spasms which are: age of 2 years or younger at time of diagnosis; seizure type consistent with spasms; presence of developmental delay or regression at the onset of spasms; and presence of EEG features of typical or atypical hypsarrhythmia.

For all cases that were identified through medical records, charts were systematically reviewed for the following data using the coding system: demographic data, seizures characteristics, developmental status, neurological examination, EEG features, neuroimaging findings, lab findings, etiology, treatment used, response to treatment, and final neurological outcome.

A total of 161 cases of infantile spasms were seen during the study period. Five cases were excluded. One case was discharged before treatment. In another case the spasms resolved spontaneously. In a third case the patient died before starting treatment and 2 cases had no follow-up after discharge.

The VGB or hormonal therapy were used in most of the cases as a first line therapy $(151 / 156)$. The VGB was the first choice in 141/156 of cases. VGB was used with an escalated dose according to response to a maximum dose of $150 \mathrm{mg} / \mathrm{kg} / \mathrm{d}$ divided twice per day. If there was no response within 2 weeks, IV synthetic ACTH (Tetracosactide) was the drug of choice, administered as an inpatient treatment. The dosing equivalent of synthetic ACTH to the natural ACTH is $0.01 \mathrm{mg}=1$ IU. The dose was chosen to give an equivalent dose to natural $\mathrm{ACTH}$ at $150 \mathrm{IU} / \mathrm{m}^{2}$ for two weeks, followed by a weaning dose given as outpatient via intramuscular route as follows: $30 \mathrm{IU} / \mathrm{m}^{2}$ daily for 3 days, $15 \mathrm{IU} / \mathrm{m}^{2}$ daily for 3 days, $10 \mathrm{IU} / \mathrm{m}^{2}$ daily for 3 days, and 10 $\mathrm{IU} / \mathrm{m}^{2}$ every other day for 3 doses, then discontinued. Deviation from this protocol was seen occasionally in the first 10 years of the study with oral steroids used sometimes with doses of $2-3 \mathrm{mg} / \mathrm{kg} / \mathrm{d}$ divided bid for 2 weeks then the dose weaned over 3 weeks until discontinuation.

Response to treatment of infantile spasms was considered complete if the spasms ceased clinically and were confirmed by video EEG and the hypsarrhythmia 
Table 1 - Comparison of responders to treatment vs non-responders groups.

\begin{tabular}{lccc}
\hline & Responders $\mathbf{n}=\mathbf{7 2}$ & $\begin{array}{c}\text { Non-responders } \\
\mathbf{n}=84\end{array}$ & P-value \\
\cline { 2 - 3 } & \multicolumn{2}{c}{$\mathbf{n}(\%)$} \\
\hline Male gender & $43(60)$ & $44(52)$ & 0.35 \\
Age(month) & & & \\
Mean (SD) & $9.7(7)$ & $9.6(6.6)$ & 0.44 \\
Etiology & & & \\
Median & 7 & 8 & \multirow{2}{*}{0.34} \\
Cryptogenic & $22(31)$ & $20(24)$ & \\
Symptomatic & $50(69)$ & $64(76)$ & \\
EEG & & & \\
Typical hypsarrhythmia & $26(36)$ & $20(24)$ & \\
Atypical hypsarrhythmia & $46(64)$ & $64(76)$ & \\
Outcome & & & \\
Fair & $14(19)$ & $0(0)$ & 0.000 \\
poor & $58(81)$ & $84(100)$ & \\
Epilepsy at 2 years & $26 / 50(52)$ & $50 / 57(88)$ & 0.000 \\
\hline
\end{tabular}

pattern resolved within one month of commencing treatment, otherwise the case was considered not responsive to treatment.

We used the West Delphi group classification of infantile spasms. ${ }^{17}$ Symptomatic is used when an identifiable cause is reached; cryptogenic is used if the case has previous developmental delay indicating a presumed underlying etiology; while idiopathic is used for cases with no clear etiology, normal development before onset of spasms, normal neurological imaging and no focality of the hypsarrhythmia.

Developmental delay was identified if one or more domains of development were delayed for the age of the patient. The severity of developmental delay is defined as mild if the functional developmental age is more than $66 \%$ for the expected age; moderate if it is between 33\% and $66 \%$ for the expected age; and severe if less than $33 \%$ for the expected age. ${ }^{18}$ The neurological outcome at follow-up was defined as fair if the patient had normal development or mild developmental delay and poor if the patient had moderate or severe developmental delay.

Tests for statistical analysis were used for both categorical and continuous variables for the group of patients with response to medical therapy versus nonresponders. The SPSS software version 24 was used for data analysis. Differences between the two groups with $p$-value of $<0.05$ was considered statistically significant.

Results. Of the 156 cases, 141 cases were treated with VGB with a complete response seen in $42(30 \%)$. The average doses in the responders compared to nonresponders were 114 and $133 \mathrm{mg} / \mathrm{kg} / \mathrm{d}$ respectively. A total of 52 cases were treated with IV Tetracosactide
(TCA) with a response in $25(48 \%)$. Of the 35 cases which initially failed VGB, 20(57\%) responded to TCA. The TCA was the first line in 8 cases with a response in $6(75 \%)$. The TCA was the third line in 8 patients with a response in $1(13 \%)$, while there was only one case treated with TCA as a fourth line with no response. There were 3 cases that were treated with a combination of TCA and VGB with complete response in all of them. Fourteen patients were treated with oral steroids with a response in 4/14(29\%). Oral steroids were used in 10 cases that failed VGB with a response in 2(20\%). Only 3 patients were treated with oral steroids as first line with a response in one. Only 4 cases were treated initially with other AEDs with a response in one treated with levetiracetam (LEV). The overall response to first line therapy was 50/156(32\%). An additional 26/156(17\%) of cases responded to second line therapy and only an additional 5/156(3\%) of the cases responded to third line therapy. Only one case responded to clonazepam after VGB failure. The overall response to treatment was $81 / 156(52 \%)$. A relapse was seen in $2 / 42(5 \%)$ of patients treated with VGB and in 5/25(20\%) of those who were treated with TCA.

The responders according to etiology were as follows: hypoxic ischemic encephalopathy $11 / 21(52 \%)$, other acquired insult 14/30(47\%); brain anomaly 4/9(44\%), genetic/syndrome 4/26(15\%), hereditary neurometabolic disorders $6 / 15(40 \%)$ and cryptogenic $15 / 35(42 \%)$. All cases with tuberous sclerosis were treated with VGB with a response of 11/13(85\%). All 7 idiopathic cases responded to treatment.

Epilepsy at 2 years was seen in 26/50(52\%) and $50 / 57(88 \%)$ of the responders and non-responders, 
respectively ( $p=0.000$ ). Only $14 / 156(9 \%)$ of the cases had a fair neurological outcome. All of them were from the responder group (Table 1 ).

Discussion. This report shows that the success of treatment in this cohort of patients with infantile spasms is $50 \%$. Synthetic ACTH shows an overall clear superiority over VGB, although it was considered a second choice in most cases. The response to oral steroids is also less favorable compared to ACTH but comparable to VGB. However, the dose of oral steroids was low. The reasons for using VGB as a first choice in the majority of cases is mainly due to the preference of families for oral medications over injections and resistance to admission. In addition, the families tend to opt not to have hormonal therapy when counseled about the potential side effects.

Aicardi et $\mathrm{al}^{19}$ proposed VGB as a first line treatment for infantile spasms in a study of 192 patients, $68 \%$ of whom had a complete response to VGB with a relapse rate of $21 \%$. Later studies showed variable responses of $26-76 \%$, with the majority around $50 \% .^{11,20}$ The response is less in symptomatic infantile spasms as compared to cryptogenic infantile spasms (29\% vs. $62 \%$ ) with an overall response of $47 \% .^{21}$ Overall, the response to VGB in our study was less than the average of other studies (30\%). The explanation for this difference is unclear. The average doses in the responders compared to non-responders were 114 and $133 \mathrm{mg} / \mathrm{kg} / \mathrm{d}$ respectively. This was comparable to the average dose in Aicardi's study of $99 \mathrm{mg} / \mathrm{kg} /$ day. ${ }^{19}$ The relapse rate is $5 \%$, which is somewhat lower than other reports of $21 \%{ }^{19}$ and $20 \% .^{22}$ The response of tuberous sclerosis patients is $85 \%$, comparable to other studies at $95 \%{ }^{23}$ and $96 \% .^{19}$

The ACTH remains the most proven, effective treatment for infantile spasms. ${ }^{24}$ Several randomized controlled trials showed efficacy of ACTH in treatment of infantile spasms with a response rate of $42-87 \%$. $^{11,12,25,26}$ This is comparable to our study with a $48 \%$ response rate to $\mathrm{ACTH}$. In a recent study, the synthetic ACTH was equally effective as the natural ACTH with responses of $70 \%$ and $81 \%$ respectively. ${ }^{27}$ The reason for the high rate of response is most likely related to using this hormonal therapy as a first line compared with our study where in most of the cases it was used as second line therapy. However, in the small number of cases in our study where ACTH was used as first line, the response was $75 \%$ which is comparable to the study by Riikonen et al. ${ }^{27}$

The response to hormonal therapy was in favor of ACTH when compared to oral steroids with a response rate of oral steroids at doses of $2-3 \mathrm{mg} / \mathrm{kg} /$ day ranging between $25-59 \% .^{25,26,28}$ This was comparable to our study at $29 \%$. However, more recent studies that tested high-dose oral steroids of $8 \mathrm{mg} / \mathrm{kg} / \mathrm{d}$ or a total of 40-60 mg per day showed a higher response rate reaching $63-80 \% .{ }^{11,12,29,30} \mathrm{~A}$ more recent randomized controlled trial studied the combination of hormonal therapy with VGB as compared with hormonal therapy alone. There was a clear benefit to the combination of both modalities. ${ }^{31}$ The 3 cases in our study that used the combination had complete responses in all of them.

The group of patients who responded to treatment have more idiopathic and tuberous sclerosis cases as compared to the non-responders group. This is comparable to other studies. ${ }^{21,24}$ Also, all patients with a fair outcome were from the responder group, while none in the non-responders group had fair outcomes. This was also true for the intractable epilepsy after the age of 2 years, with a higher number of cases of the non-responder group developing epilepsy. Mohamed et al found at one year of follow-up of infantile spasms, 31/40(77.5\%) continued to have seizures. ${ }^{32}$ Of the responders, $11 / 18(61 \%)$ and the non-responders, 20/22(91\%), had epilepsy at follow-up. ${ }^{32}$ These findings are comparable with our study of $71 \%$ epilepsy at follow-up, including $52 \%$ and $88 \%$ of the responders and non-responders, respectively. With regard to developmental outcomes in a study by Karvelas et al ${ }^{22} 17 / 72(23 \%)$ of patients had good outcomes. Of the symptomatic infantile spasms, $1 / 42(2 \%)$ had good outcomes and of the cryptogenic infantile spasms, 16/30(53\%) had good outcomes..22 Recovery was seen only in patients who responded completely to therapy. ${ }^{22}$ Good outcome was seen in 9\% in our study; all of them had a complete response to treatment.

The main limitation of this study is its retrospective chart review nature and small sample size. However, the results reflect treatment of infantile spasms in real-life practice and the report of one center's experience in a relatively large cohort of patients. The study gives insight into considering hormonal therapy use as first line in this part of the world where it is still underutilized. Also, the study is the first of its kind where intravenous synthetic ACTH was the route of treatment. Clearly, multicenter randomized trials are desirable to study new modalities of therapies as the overall response to current modalities is still suboptimal and the outcome is still unfavorable. Also, there is a need for studies on novel therapies that target the pathophysiology and underlying etiology of this serious epilepsy syndrome.

Conclusion. The response to VGB is suboptimal, while the response to synthetic ACTH is encouraging making it a good alternative for the costly and hard-toobtain natural ACTH as a potential first line therapy in infantile spasms. This is particularly important in the subgroup of patients with cryptogenic and idiopathic etiology. 
Acknowledgement. The authors would like to to thank. www.englishtrackers.com for English language editing.

\section{References}

1. Chudomelova L, Morris H. Scantlebury, Raffo E, Coppola A, Betancourth D, et al. Modeling new therapies for infantile spasms. Epilepsia, 2010; 51 Suppl 3: p. 27-33.

2. Wong M, Trevathan E. Infantile spasms. Pediatr Neurol 2001; 24: 89-98.

3. Riikonen, R. The latest on infantile spasms. Curr Opin Neurol 2005; 18: 91-95.

4. Lagae L, Verhelst H, Ceulemans B, Meirleir LD, Nassogne MG, Borchgrave VD, et al. Treatment and long term outcome in West syndrome: the clinical reality. A multicentre follow up study. Seizure 2010; 19: 159-164.

5. Proposal for revised classification of epilepsies and epileptic syndromes. Commission on Classification and Terminology of the International League Against Epilepsy. Epilepsia 1989; 30: 389-399.

6. Caraballo R, Cersósimo R, Intruvini S, Pociecha J, Fejerman $\mathrm{N}$. [West's syndrome in patients with cerebral paralysis and periventricular leukomalacia: a good response to treatment]. Rev Neurol 1997; 25: 1362-1364. Spanish

7. Alrifai MT, AlShaya MA, Abulaban A, Alfadhel M. Hereditary neurometabolic causes of infantile spasms in 80 children presenting to a tertiary care center. Pediatr Neurol 2014; 51: 390-397.

8. Al-Abdulkareem AA, Ballal SG. Consanguineous marriage in an urban area of Saudi Arabia: rates and adverse health effects on the offspring. J Community Health 1998; 23: 75-83.

9. El-Mouzan MI, Al-Salloum AA, Al-Herbish AS, Qurachi $\mathrm{MM}, \mathrm{Al}-\mathrm{Omar} \mathrm{AA}$. Regional variations in the prevalence of consanguinity in Saudi Arabia. Saudi Med J 2007; 28: 1881-1884.

10. Elterman RD, Shields WD, Mansfield KA, Nakagawa J, US Infantile Spasms Vigabatrin Study Group. Randomized trial of vigabatrin in patients with infantile spasms. Neurology 2001; 57: 1416-14121.

11. Lux AL, Edwards SW, Hancock E, Johnson AL, Kennedy CR, Newton RW. The United Kingdom Infantile Spasms Study (UKISS) comparing hormone treatment with vigabatrin on developmental and epilepsy outcomes to age 14 months: a multicentre randomised trial. Lancet Neurol 2005; 4: 712-717.

12. Kossoff EH, Hartman AL, Rubenstein JE, Vining EPG. High-dose oral prednisolone for infantile spasms: an effective and less expensive alternative to ACTH. Epilepsy Behav 2009; 14: 674-676.

13. Mackay MT, Weiss SK, Adams-Webber T, Ashwal S, Stephens D, Ballaban-Gill K, et al. Practice parameter: medical treatment of infantile spasms: report of the American Academy of Neurology and the Child Neurology Society. Neurology 2004; 62: 1668-1681.

14. Jaseja H. Drug-choice in management of West syndrome (infantile spasms): Early ACTH treatment may offer a better prognostic outcome. Medical hypotheses 2008; 70: 197-198.

15. Riikonen R. Infantile Spasms: Outcome in Clinical Studies. Pediatr Neurol 2020; 108: 54-64.

16. O'Callaghan FJ, Lux AL, Darke K, Edwards SW, Hancock E, Johnson AL. The effect of lead time to treatment and of age of onset on developmental outcome at 4 years in infantile spasms: evidence from the United Kingdom Infantile Spasms Study. Epilepsia 2011; 52: 1359-1364.
17. Lux AL, Osborne JP. A proposal for case definitions and outcome measures in studies of infantile spasms and West syndrome: consensus statement of the West Delphi group. Epilepsia 2004; 45: 1416-1428.

18. Shevell MI, Majnemer A, Rosenbaum P, Abrahamowicz M. Etiologic determination of childhood developmental delay. Brain \& development 2001; 23: 228-235.

19. Aicardi J, Mumford JP, Dumas C, Wood S. Vigabatrin as initial therapy for infantile spasms: a European retrospective survey. Sabril IS Investigator and Peer Review Groups. Epilepsia 1996; 37: 638-642.

20. Nelson GR. Management of infantile spasms. Transl Pediatr 2015; 4: 260-270.

21. Fejerman N, Cersósimo R, Caraballo R, Grippo J, Corral $\mathrm{S}$, Martino RH, et al. Vigabatrin as a first-choice drug in the treatment of West syndrome. J Child Neurol 2000; 15: 161-165.

22. Karvelas G, Lortie A, Scantlebury MH, Duy PT, Cossette P, Carmant L. A retrospective study on aetiology based outcome of infantile spasms. Seizure 2009; 18: 197-201.

23. Hancock E, Osborne JP. Vigabatrin in the treatment of infantile spasms in tuberous sclerosis: literature review. J Child Neurol 1999; 14: 71-74.

24. Go CY, Mackay MT, Weiss SK, Stephens D, Adams-Webber T, Ashwal S, et al. Evidence-based guideline update: medical treatment of infantile spasms. Report of the Guideline Development Subcommittee of the American Academy of Neurology and the Practice Committee of the Child Neurology Society. Neurology 2012; 78: 1974-1980.

25. Hrachovy RA, Frost Jr JD, Kellaway P, Zion TE. Double-blind study of ACTH vs prednisone therapy in infantile spasms. $J$ Pediatr 1983; 103: 641-645.

26. Snead OC, 3rd, Benton JW, Myers GJ. ACTH and prednisone in childhood seizure disorders. Neurology 1983; 33: 966-970.

27. Riikonen R, Lahdetie J, Kokki H. ACTH Treatment of Infantile Spasms: Low-Moderate- Versus High-Dose, Natural Versus Synthetic ACTH-A Retrospective Cohort Study. Pediatr Neurol 2020; 111: 46-50.

28. Baram TZ, Mitchell WG, Tournay A, Snead OC, Hanson RA, Horton EJ. High-dose corticotropin (ACTH) versus prednisone for infantile spasms: a prospective, randomized, blinded study. Pediatrics 1996; 97: 375-379.

29. Hussain SA, Shinnar S, Kwong G, Lerner JT, Matsumoto JH, Wu JY, et al. Treatment of infantile spasms with very high dose prednisolone before high dose adrenocorticotropic hormone. Epilepsia 2014; 55: 103-107.

30. Eliyan Y, Heesch J, Alayari A, Rajaraman RR, Sankar R, Hussain SA. Very-High-Dose Prednisolone Before ACTH for Treatment of Infantile Spasms: Evaluation of a Standardized Protocol. Pediatr Neurol 2019; 99: 16-22.

31. O'Callaghan FJK, Edwards SW, Alber FD, Hancock E, Johnson AL, Kennedy CR. Safety and effectiveness of hormonal treatment versus hormonal treatment with vigabatrin for infantile spasms (ICISS): a randomised, multicentre, open-label trial. Lancet Neurol 2017; 16: 33-42.

32. Mohamed BP, Scott RC, Desai N, Gutta P, Patil S. Seizure outcome in infantile spasms--a retrospective study. Epilepsia 2011; 52: 746-752. 